\title{
Der jordanisch-israelische Friedensvertrag
}

\author{
Von Irene Schlünder und Ferhad Ibrahim
}

\section{Einleitung}

Die feierliche Unterzeichnung des Friedensvertrages zwischen dem Haschemitischen Königreich Jordanien und Israel ${ }^{1}$ ist für das bilaterale Verhältnis der Vertragsparteien weniger spektakulär, als es zunächst erscheinen mag. Denn de facto herrscht schon seit geraumer Zeit eine Art "kalter Frieden". Dies drückt sich aus in wiederholten hochrangigen Begegnungen zwischen beiden Seiten, wenn sie auch wegen der äußeren Situation oft diskret behandelt wurden. ${ }^{2}$ Der Vertragsschluß berührt indessen nicht allein das Verhältnis zwischen den Parteien, sondern ist neben dem Gaza-Jericho-Abkommen ein weiterer grundlegender Schritt für den Friedensprozeß im Nahen Osten, und die israelisch-jordanischen Verhandlungen waren eng verzahnt mit dem Fortgang des Friedensprozesses zwischen Israel und den Palästinensern. Betroffen sind ferner die jordanisch-palästinensischen Beziehungen. Jordanien, das seit 1950 die Westbank als eigenes Staatsgebiet beansprucht hatte, hatte schon in den siebziger Jahren angefangen, Schritt für Schritt die politische Verantwortung für die Westbank den Palästinensern zu überlassen. Dieser Prozeß begann 1974 mit der Anerkennung der PLO als der einzigen legitimen Vertreterin der Palästinenser. Schließlich verzichtete Jordanien im Juli 1988 auf seine administrative Zuständigkeit für das Westjordanland. Damit beendete es seine politische und rechtliche Verantwortung für die Westbank. Die Ausrufung des Staates Palästina durch den Palästinensischen Nationalrat auf seiner Sitzung in Algier im November 1988 erweckte sogar den Eindruck, daß Jordanien mit seinem Schritt den Weg für die Etablierung eines unabhängigen palästinensischen Staates freimachen wollte.

Erleichtert wurde die Unterzeichnung des Vertrages mit Israel durch die Tatsache, daß Jordanien, anders als Ägypten und Syrien, keine Ambitionen auf regionale Hegemonie hatte, die eine zusätzliche Dimension des arabisch-israelischen Konflikts ausmacht. Jordanien hatte jedoch spezifische Gründe für die Beilegung des Konflikts mit Israel. Diese lagen in der besonderen Vorgeschichte und Entwicklung des Staates Jordanien. Obwohl Jordanien nach dem verlorenen Krieg von 1967, der ihm nicht nur den Verlust der West-

Text in: International Legal Materials, Vol. XXXIV (1995), S. 46-66 (maßgebliche engl. Fassung), und in: Internationale Politik [ehem. Europa-Archiv], 50. Jg. (1995), S. 86-109 (dt. Übers.).

Vgl. zu den diskreten israelisch-jordanischen Begegnungen zwischen 1948 und 1993 den Bericht der Süddeutschen Zeitung vom 26. Juli 1994. 
bank samt Jerusalem, sondern darüber hinaus Ströme von Flüchtlingen aus dem Westjordanland bescherte, den Wunsch hatte, den Konflikt mit Israel beizulegen ${ }^{3}$, konnte es anders als Ägypten keinen Separatfrieden mit Israel schließen. Dies lag nicht nur an dem geringen Gewicht des ressourcenarmen Jordanien in der regionalen Politik - denn dies hätte durch die Zusammenarbeit mit Ägypten und durch westliche politische und ökonomische Unterstützung kompensiert werden können -, sondern eher an der demographischen Struktur Jordaniens mit seiner palästinensischen Mehrheit. ${ }^{4}$ Für das jordanische Königreich ist der Friedensvertrag insofern von Bedeutung, als die politische Legitimation des Haschemitischen Königshauses nicht nur von den radikalen palästinensischen Gruppen bestritten wurde, sondern seit der Machtübernahme durch den Likud-Block in Israel auch durch die rechten Israelis. Letztere vertraten offen die These, daß Jordanien ein Palästinenser-Staat sei und es keinen Bedarf für einen zweiten palästinensischen Staat gebe. Mit dieser These versuchten die rechten israelischen Parteien, die beabsichtigte Annexion des Westjordanlandes zu rechtfertigen, das im Sprachgebrauch dieser Parteien Judäa und Samaria heißt.

Auch wenn Jordanien essentielles Interesse daran hatte, seine Beziehungen mit Israel vertraglich zu regeln, waren die Bedingungen vor dem zweiten Golfkrieg für Verhandlungen noch nicht gegeben. Erst die regionale Entwicklung während und nach der KuwaitKrise ermöglichte die Verhandlungen zwischen Israel und seinen Nachbarn. Die Rolle Jordaniens bei den Verhandlungen seit der Madrider Nahostkonferenz war dadurch kompliziert, daß sie mit der der Palästinenser verflochten war, und nicht etwa, weil die Lösung des jordanisch-israelischen Konfliktes schwierig gewesen wäre. Die Regierung Schamir weigerte sich aus politischen und legitimatorischen Gründen, mit einer selbständigen palästinensischen Delegation zu verhandeln. So konnten die palästinensischen Politiker nur als Mitglieder der jordanischen Delegation an Nahost-Verhandlungen teilnehmen.

Spätestens als Anfang 1993 die Friedensverhandlungen ins Stocken gerieten, wurde deutlich, daß ein Durchbruch durch ein Splitting der Konflikte erzielt werden konnte. Allerdings mußte Jordanien sich mit Syrien koordinieren, um einen Konflikt mit seinem arabischen Nachbarland zu vermeiden. Aber wegen der Verzahnung des syrisch-israelischen Konfliktes mit der Politik und Präsenz Israels und Syriens im Libanon war den Jordaniern bewußt, daß die Verhandlungen zwischen Syrien und Israel eine geraume Zeit beanspruchen würden. ${ }^{5}$

3

Vgl. hierzu das Buch des jordanischen Kronprinzen al-Hasan bin Talal, Al-Sai nahwa al-salam (Das Streben nach Frieden), Amman 1985, S. 125 ff.

4

Vgl. hierzu Shirin H. Fathi, Jordan, an invented Nation, Hamburg, Deutsches Orient-Institut 1994.

5

Jwad al-Inani, Mitglied der jordanischen Verhandlungsdelegation mit Israel, erklärte in einem Interview mit der jordanischen Tageszeitung al-Aswaq, daß Jordanien, wenn es den Vertrag mit 
Der Abschluß der "Declaration of Principles on Interim Self-Government Arrangements" ${ }^{6}$, der nach geheimen palästinensisch-israelischen Verhandlungen in Oslo verabschiedet worden war und am 13. September 1993 in Washington unterzeichnet wurde, gab Jordanien endlich den Weg frei, die Verhandlungen mit Israel voranzutreiben. Im wesentlichen konzentrierten sich die Delegationen Israels und Jordaniens seit Januar 1994 auf die Erzielung eines Kompromisses hinsichtlich zweier Fragen, die den Friedensschluß zwischen den beiden Staaten noch behinderten: die Anerkennung der Grenzen zwischen Israel und Jordanien, die vom Völkerbund bei Erteilung des Mandates über Palästina an Großbritannien festgelegt worden waren, und die Regelung der Nutzungsteile an den Flüssen Jordan und Yarmuk. Andere Fragen, die wegen ihrer regionalen Interdependenz bilateral nicht gelöst werden konnten (die Flüchtlingsfrage, die regionale ökonomische Zusammenarbeit und regionale Sicherheit), konnten nur in Umrissen behandelt werden. Die Washingtoner Erklärung, die am 25. Juli 1994 zwischen König Hussein und dem israelischen Ministerpräsidenten Rabin unterzeichnet wurde und die den ersten wichtigen Durchbruch in den Verhandlungen zwischen den beiden Staaten darstellte, befaßte sich denn auch lediglich mit bilateralen Fragen. ${ }^{7}$ So war durch die Unterzeichnung dieser Erklärung der Weg zum Friedensvertrag vom Oktober 1994 zwischen den beiden Staaten geebnet. Die Stabilität des Friedens zwischen Jordanien und Israel ist allerdings weiterhin abhängig von den Faktoren, die ausgeklammert wurden, wie der Lösung des Flüchtlingsproblems, der regionalen wirtschaftlichen Zusammenarbeit sowie der regionalen Sicherheit. Anders als bei der Lösung der territorialen Streitigkeiten können diese Fragen ohne multilaterale Verhandlungen und internationale Unterstützung kaum gelöst werden.

In diesem Beitrag untersuchen wir im ersten Teil die wirtschaftlichen und innenpolitischen Determinanten der jordanischen Friedenspolitik. Im zweiten Teil des Beitrags sollen das Vertragswerk und die damit verbundenen Probleme untersucht werden.

\section{Die innenpolitischen und wirtschaf tlichen Determinanten der jordanischen Friedenspolitik}

Die nach der Unterzeichnung der Washingtoner Erklärung zwischen Israel und Jordanien durchgeführte repräsentative Befragung zeigte überraschend, daß 80,2 Prozent der Befragten den Friedensschluß mit Israel befürworteten und daß nur 14,1 Prozent der Befragten

Israel nicht geschlossen hätte, die regionale Hegemonie Syriens anerkannt hätte. Siehe al-Aswaq vom 26. Oktober 1994. 
den Frieden ablehnten, während 5,7 Prozent sich dazu nicht äußerten. ${ }^{8}$ Dieses Ergebnis war um so erstaunlicher, als die jordanischen Islamisten, die eine ablehnende Haltung zum Frieden mit Israel zeigten ${ }^{9}$, bei den Parlamentswahlen von 1989 immerhin 32 der 80 Mandate erlangten. Bei den Wahlen von 1993 sank allerdings die Zahl der Abgeordneten der Islamischen Aktionsf ront, ein Bündnis der Muslimbruderschaft mit den kleineren islamistischen Gruppen, auf 21 Mandate. ${ }^{10}$ Um den offensichtlichen Widerspruch zwischen der Haltung der Bevölkerung zum Friedensprozeß und ihrem Verhalten bei den Wahlen zu erklären, soll hier kurz die Grundstruktur des jordanischen wirtschaftlichen und politischen Systems umrissen werden.

\section{Wirtschaftliche Determinanten}

Der jordanische Staat war seit seiner Gründung 1921 ein Rentierstaat ${ }^{11}$, der angesichts seiner schwachen ökonomischen Strukturen und kaum vorhandenen strategischen Ressourcen, wie etwa Erdöl, auf ausländische Zuwendungen und seit den fünfziger Jahren zusätzlich auf Überweisungen der Gastarbeiter in den arabischen Golfstaaten angewiesen ist. ${ }^{12} \mathrm{Da}$ sich Jordanien nur geringfügig auf eigene rentenerbringende Ressourcen stützen kann - vor allem Phosphat und Pottasche, die etwa 35 Prozent des gesamten Exportvolumens Jordaniens ausmachen ${ }^{13}$ - unterlag es starken Schwankungen, die für die Rentenarten, die es bezog, charakteristisch sind. So steckte Jordanien Ende der achtziger Jahre in einer ernsthaften Wirtschaftskrise wegen des Sinkens der Zuwendungen aus den arabischen Golfstaaten infolge der regionalen Rezession sowie der Verringerung des Transithandelsvolumens mit dem Irak nach dem Waffenstillstand im Krieg zwischen Iran und Irak. Der jordanische

Vgl. Center for Strategic Studies, University of Jordan: Istitla al-Rai hawla al-mufawadat alurduniya al-israiliya (Meinungsbefragung über die jordanisch-israelischen Verhandlungen), Amman 1994.

Zu den jordanischen Islamisten vgl. Samih al-Muaita, llan Washington fi al-mizan (Die Erklärung von Washington auf dem Prüfstand), Amman 1994.

$\mathrm{Zu}$ ausführlichen Angaben über die Wahlen von 1993 vgl. Hubert Dobers, Die Wahlen zum zwölften Unterhaus Jordaniens. Analyse der Ergebnisse und erste Entwicklungen, in: KonradAdenauer-Stiftung-Auslandsinformationen, 10. Jg., Nr. 2. 1994, S. 32-46.

11 Rentierstaaten sind nach Peter Pawelka "jene Staaten der Dritten Welt, die regelmäßig in sehr hohem Umfang externe Rente oder Renten-Äquivalente beziehen und deren Verhalten als Funktion der Struktur dieser besonderen Staatsrevenuen und des mit diesem Einkommen betriebenen Politikmanagements nach innen begriffen werden kann." Peter Pawelka, Der Vordere Orient und die internationale Politik, Stuttgart usw.: Kohlhammer 1993, S. 103.

Zur ökonomischen Situation Jordaniens vor dem Friedensvertrag siehe: Hani Abu-Jabarah, The Economics of Peace: Jordan, in: Stanley Fischer / Dani Rodrik / Elias Tuma, The Economics of Middle East Peace, Cambridge, Massachusetts 1992, S. 181-199.

Vgl. Alan J. Day, Jordan: Economy, in: The Middle East and North Africa, 1995. Forty-First Edition, London 1994, S. 588. 
König erklärte auf der arabischen Gipfelkonferenz von Bagdad im Mai 1990 unverhohlen, daß sein Land vor dem Bankrott stünde, falls die arabischen Erdölstaaten keine baldige Finanzhilfe leisteten $^{14}$. Tatsächlich verschuldete sich Jordanien seit Mitte der achtziger Jahre hoch. 1989 wurden die Auslandsschulden - verursacht u.a. durch das chronische Haushaltsdefizit - mit 6.467 Mio. US \$ beziffert; das Land mußte im gleichen Jahr 495 Mio. US \$ an Zinsen aufbringen. 1993 hatte Jordanien 7.184 Mio. US \$ Auslandsschulden und mußte eine Summe von 517 Mio. US \$ für den Schuldendienst ausgeben. ${ }^{15} \mathrm{Zwar}$ zog der zweite Golfkrieg Jordanien in vieler Hinsicht in Mitleidenschaft ${ }^{16}$, vor allem durch Flüchtlingsströme der jordanischen und palästinensischen Gastarbeiter aus den Golfstaaten sowie durch die ausbleibende finanzielle Hilfe aus den Golfstaaten und das völlige Erliegen des Handels mit dem Irak. Doch zeigte die Wirtschaft dank des Kapitalzuflusses durch die heimkehrenden Gastarbeiter eine bis 1993 anhaltende Konjunktur. So erlebte Jordanien von 1991 bis Ende 1993 einen "Miniboom". ${ }^{17}$ Die Wachstumsrate der jordanischen Wirtschaft wurde im genannten Zeitabschnitt auf etwa 5,0 Prozent beziffert ${ }^{18}$. Dieser Wirtschaftsaufschwung kam bereits Ende 1993 zum Stillstand, weil die Konjunktur im Bausektor, wo die 1990 heimkehrenden Gastarbeiter ihr Kapital vor allem investiert hatten, abflaute. Da das Ende des UNO-Embargos gegen den Haupthandelspartner Jordaniens, den Irak, nicht in Sicht war und die arabische Unterstützung für Jordanien wegen der jordanischen Position im Golfkrieg ausblieb, mußte Jordanien versuchen, verstärkt Zugang zu externer Rente zu suchen. Nachdem Ägypten durch den Abschluß des Camp-DavidAbkommen 1978/1979 nicht unerheblich mit westlicher Hilfe bedacht worden war, erhoffte sich Jordanien ebenfalls eine großzügige westliche Friedensrente. ${ }^{19}$ Dieser Grund erhöhte die Bereitschaft Jordaniens, mit Israel Frieden zu schließen.

14

Zum vollständigen Text der Rede König Husseins vgl. die kuwaitische Tageszeitung al-Qabas vom 29. Mai 1990.

Die Schulden Jordaniens. wuchsen bis 1993 auf etwa 7 Mrd. US \$. Jordanien mußte mit den Geberstaaten (Pariser Club) und mit den Privatgläubigem (Londoner Club) immer wieder Umschuldungsverhandlungen führen, siehe hierzu World Debt Tables, Washington 1994; Thomas Koszinowski/Hanspeter Mattes, Nahost-Jahrbuch 1993. Leverkusen, Leske u. Budrich, 1994.

Vgl. zur Position Jordaniens während des zweiten Golfkrieges: Kamel Abu Jaber, Jordan and the Gulf War, in: Tareq Y. Ismael / Jaqueline S. Ismael (Hrsg.), The Gulf: War and the New World Order, Gainesville usw., Univ. Pr. of Florida, S. 366-382.

Nahost-Jahrbuch 1992, S. 103.

Ebd. und das Nahost-Jahrbuch 1993, S. 104.

Vgl. George Hawatmeh, Why the King signed. In Middle East International vom 5. August 1994, S. 4. 


\section{Innenpolitische Determinanten}

Die ökonomische Struktur Jordaniens steht in einer reziproken Relation zum innenpolitischen System. Nach innen baute die Haschemitische Dynastie ein Klientelsystem auf, das der Monarchie trotz zahlreicher interner und externer Konflikte Stabilität verlieh. Repräsentanten der Stämme, der ethnischen Minderheiten, der religiösen Gemeinschaften, aber auch der wichtigsten sozialen Gruppen, etwa der Händlerschicht, bilden mit der Dynastie ein Netz. Die Loyalität zur Monarchie wird durch politische Partizipation und Zugang zum Rentenzyklus honoriert. Daß dieses System fragil ist, wird in den Krisenzeiten sehr deutlich. Die Wirtschaftskrise Ende der achtziger Jahre führte 1989 zu gefährlichen Unruhen in Südjordanien, die durch Gewalt und Versprechungen abgewendet werden konnten. ${ }^{20}$ Die jordanische Führung stand nach den Unruhen vor der Option, entweder nach dem Beispiel der anderen arabischen Staaten die Opposition dauerhaft gewaltsam zu unterdrücken oder eine kontrollierte politische Partizipation der oppositionellen Kräfte zu ermöglichen. Die Opposition der achtziger Jahre war islamistisch orientiert. ${ }^{21}$ Arabische Nationalisten und Linke verloren seit den siebziger Jahren rapide an Einfluß und waren nun eine Randerscheinung. Eine Besonderheit der jordanischen islamistischen Opposition lag darin, daß sie seit den fünfziger Jahren ein Zweckbündnis mit der haschemitischen Monarchie eingegangen war. Dieses Bündnis war gegen die gemeinsamen Feinde, die arabischen Nationalisten und die Linke, gerichtet. Dies war auch der Grund dafür, daß die Islamisten die Monarchie weitestgehend von ihrer Kritik ausklammerten.

Ein anderes Problemfeld ergab sich aus der Frage der jordanischen Identität, wenngleich diese lange Zeit nicht offen diskutiert wurde. Die Frage rückte stetig ins Zentrum des politischen Diskurses, nachdem Jordanien sich politisch und rechtlich vom Palästina-Problem abgekoppelt hatte. In Jordanien war es seit den fünf ziger Jahren zur festen Regel geworden, daß die sensiblen Positionen in der Armee, bei den Sicherheitskräfte und in der Verwaltung mit Jordaniern nicht-palästinensischer Herkunft besetzt wurden. Die Zugang für die Palästinenser zu höheren Ämtern war aber nicht völlig verschlossen. Nicht selten bekleideten und bekleiden die mit der Monarchie verbundenen Personen palästinensischen Ursprungs verantwortungsvolle Positionen im Staat. Die Frage, die sich nach der Unterzeichnung der palästinensisch-israelischen "Declaration of Principles" stellte, bezog sich vielmehr darauf, welche Identität die Palästinenser jordanischer Staatsbürgerschaft haben. Unter dem Motto

Die Unruhen vom Frühjahr 1989 brachen im ökonomisch schwachen Süden aus. Dort traf der vom Internationalen Währungsfond (IWF) empfohlene Abbau von Lebensmittelsubventionen die Bevölkerung noch härter als im besser entwickelten Norden des Landes, vgl. hierzu den Bericht der Neuen Zürcher Zeitung vom 25. April 1989.

Zum Islamismus in Jordanien vgl. Iyad al-Barghuthi, Al-Islam baina al-Sulta wa al-Mu`arada fi al-Urdun (Der Islam zwischen der Regierung und der Opposition in Jordanien), in: Mahmud Amin al-Alim, Al-Islam al-Siyasi (Der politische Islam), Kairo, Dar al-thaqafa al-Jadida, 1989, S. 234241. 
"Konsolidierung der jordanischen Identität" (ta ziz al-hawiya al-urduniya) führte die halboffiziöse jordanische Presse seit Anfang 1994 offen einen Diskurs über das Thema der Identität. ${ }^{22}$ Hier wurde deutlich, daß nach dem israelisch-palästinensischen Friedensvertrag nicht ganz unbegründete Unsicherheiten über das Verhalten der jordanischen Palästinenser entstanden. Es geht hier um diejenigen Palästinenser, die bereits vor 1967 in Jordanien ansässig waren, da davon ausgegangen wird, daß diese so weit integriert sind, da $\beta$ die Frage ihrer Rückkehr in ihre angestammten Gebiete nur noch theoretischer Natur oder eine Frage von Entschädigungsregeln ist. Aber selbst diejenigen Palästinenser, die 1967 nach Jordanien geflohen sind und deren Rückkehr noch ernsthaft zur Debatte steht, werden, wie König Hussein nach der Unterzeichnung der Washingtoner Erklärung nahelegte ${ }^{23}$, auch nach einer entsprechenden Rückkehrregelung zwangsweise repatriiert. Daß die Entscheidung über die Identität nicht dem Zufall überlassen wurde, zeigt das 1993 vom neuen Parlament verabschiedete Wahlgesetz, das die Wahlbezirke neu aufteilt. ${ }^{24}$ Nach dem Wahlgesetz Nr. 22 von 1986 und der Novellierung von 1993 wurden die dünnbesiedelten, von Ost jordaniern bewohnten Südbezirke bezüglich der Zahl der zu wählenden Abgeordneten den nördlichen, dichtbesiedelten Wahlbezirke mit einer palästinensischen Mehrheit gleichgestellt. So kamen in dem Wahlbezirk Amman auf 220.000 drei zu wählende Abgeordnete, während im südjordanischen Wahlbezirk al-Tufail 24.000 Wähler die gleiche Anzahl von Kandidaten wählen. ${ }^{25}$ Durch das Gesetz wurden zwei Ziele erreicht: politische Marginalisierung der Islamisten und zugleich die der Palästinenser, denn es war bekannt, daß die führenden Politiker der islamistischen Gruppen, aber auch deren Anhänger vorwiegend Palästinenser sind. Ein zweiter wichtiger Aspekt des Gesetzes von 1993 ist die Abschaffung der Stimmhäufung; während nach dem Gesetz von 1986 der Wähler so viele Stimmen hatte wie die Zahl der zu wählenden Abgeordneten in seinem Wahlbezirk, darf er nunmehr nur einen einzigen Kandidaten wählen. Das Stimmenpotential der Islamisten wurde dadurch beeinträchtigt, da die Islamisten bei den für sie erfolgreichen Wahlen von 1989 Wahlbündnisse mit den lokalen Notabeln, Stämmen und kleineren Parteien eingegangen waren, was unter den veränderten Umständen nicht mehr opportun ist. Die Frage, ob das politische System dadurch mehr an Stabilität gewonnen hat, kann hier nicht abschließend beantwortet werden. Jedenfalls zeigten die Unruhen von 1989, die sich in der rein jordanischen südlichen Region ereigneten, daß die Frage der Identität allein kein entscheidender Faktor für

So z.B. der führende jordanische Journalist Fahd al-Fanik in seinen Leitartikeln in der Zeitung alDastur September/Oktober 1994.

König Hussein äußerte sich in einem Interview mit "Der Spiegel" folgendermaßen: "Jedes Mitglied der jordanischen Gemeinschaft behält seinen Status bis zu dem Tag, an dem sich die Möglichkeit bietet, über den weiteren Verbleib im jordanischen Staatsverband zu entscheiden", in: Der Spiegel 34/1994. 
die Stabilität ist. Davon abgesehen versuchte König Hussein, den Demokratisierungsprozeß voranzutreiben, der mit den Parlamentswahlen von 1989 seinen Anfang gefunden hatte, als nach dem zweiten Golfkrieg die Signale darauf deuteten, daß der Westen und vor allem die USA die Verhandlungen zwischen Israel und seinen Nachbarn im Interesse der Stabilität im Nahen Osten beschleunigen würden. Die am 9.6.1991 verkündete Nationalcharta (alMithaq al-Watani) brachte tatsächlich den Demokratisierungsprozeß des Landes voran ${ }^{26}$ : Zwar blieb die Stellung des Königs, wie sie in der Verfassung verankert ist, unangetastet ${ }^{27}$; König Hussein war jedoch bereit, den Ausnahmezustand zu beenden und die politischen Parteien zuzulassen, die bis dahin zwar nicht alle verboten waren, aber am Prozeß der politischen Willensbildung nicht offiziell teilnehmen konnten. ${ }^{28}$ Sicherlich versuchte König Hussein durch die eingeleiteten Schritte zur Demokratisierung des Systems, die Monarchie politisch zu legitimieren, aber diese Schritte stehen in engem Kontext mit dem Friedensprozeß. Ein demokratisches Jordanien sollte als Modell für die anderen arabischen Staaten dienen. ${ }^{29}$ Der Demokratisierungsprozeß darf aber nicht darüber hinwegtäuschen, $\mathrm{da}$ der König gestützt auf die Verfassung die politische Entwicklung im Land weiter unter seiner Kontrolle hält. So entließ der König im Januar 1995 den bisherigen Ministerpräsidenten Agd al-Salam al Majali, als offensichtlich wurde, daß die Friedensdividenden, die von den westlichen Staaten geleistet worden waren, weit unter den jordanischen Erwartungen lagen. Daß die USA für das Jahr 1995 lediglich 225 Mio. US \$ jordanische Schulden streichen würden, wurde von König Hussein als nicht sehr ermutigend für den Friedensprozeß betrachtet. ${ }^{30}$ Zudem konnte die Regierung al-Majali die Beziehungen mit den arabischen Golfstaaten, abgesehen von Katar und Oman, nicht normalisieren. Für die Bewältigung der Wirtschaftskrise und die Regelung der palästinensisch-jordanischen Beziehungen

Siehe zum vollständigen Text der Nationalcharta: Risalat Majlis al-Umma: Al-Mithaq al-Watani (Mitteilungsbrief des Nationalrates: Die Nationalcharta), August/Oktober 1991.

In der 1952 verkündeten jordanischen Verfassung erhält der Monarch weitgehende Befugnisse (Art. 28-40): Er löst das Parlament auf, ernennt die Mitglieder des Senats (Majlis al-A iyan) und deren Präsidenten und ernennt den Ministerpräsidenten. Er ist der oberste Befehlshaber der Streitkräfte; er erklärt den Krieg und schließt Frieden sowie Verträge mit anderen Staaten. Vgl. Majmu`at al-Dastur al-Urduni (Sammelblatt der jordanischen Verfassung), Amman: al-Jadida li al-Tiba`a 1990, S. 17-24.

Ende 1994 waren über 30 Parteien, einschließlich die Kommunistische Partei Jordanien und die islamistische Muslimbruderschaft, zugelassen.

So ließ König Hussein öffentlich im jordanischen Fernsehen seinen bis zum zweiten Golfkrieg engen Verbündeten Saddam Hussein wissen, daß Jordanien eine Demokratisierung des Irak als wichtige Prämisse zur Beendigung des 1990 verhängten Embargos sieht, vgl. Iraqi File, Nr. 13, 1993, S. 15.

30

Vgl. George Hawtmeh, Frustrated on several counts, in: Middle East International vom 17. März 1995, S. 6-7. Für das Jahr 1996 plante der amerikanische Kongreß eine Unterstützung für Jordanien in der Höhe von nur 48 US \$, ebenda., S. 6. 
ernannte der König seinen Vetter Sharif Zaid bin Shakir, einen in der Armee einflußreichen ehemaligen Offizier, zum Ministerpräsidenten. ${ }^{31}$

Die besonderen politischen Strukturen, vor allem der Klientelismus und die nach dem Beginn des Friedensprozesses aufgekommene jordanische Identität, schufen tatsächlich günstige Bedingungen für die Akzeptanz des Friedens mit Israel. Die islamistische Opposition sowie die nationalistischen und linken Parteien (Islamische Aktionsfront, die BaathPartei und die Kommunisten), die zusammen im Parlament nur 20 Sitze haben, hatten nicht nur keine Möglichkeit, die Ratifizierung des Friedensvertrags im Parlament zum Scheitern zu bringen ${ }^{32}$, sondern darüber hinaus blieben ihre außerparlamentarischen Protestaktionen fast ohne Wirkung. ${ }^{33}$ Angesichts der sehr zurückhaltenden finanziellen Hilfe aus dem Westen und der immer noch gestörten Beziehungen mit den arabischen Golfstaaten, allen voran Saudi-Arabien, hatte Jordanien gehofft, durch eine enge ökonomische Zusammenarbeit mit Israel seine Wirtschaftsprobleme zu bewältigen. ${ }^{34}$ Der Friedensvertrag strebt die völlige Normalisierung der Wirtschaftsbeziehungen zwischen den beiden Staaten an. Da der Nahostmarkt, eine Art Middle East Community, ${ }^{35}$ eine endgültige Beilegung des arabisch-israelischen Konflikts und die regionale Zusammenarbeit aller nahöstlichen Staaten voraussetzt, scheint er eher eine Vision für die Zukunft zu sein. ${ }^{36}$ Eine Lösung des Palästinenser-Problems und ein funktionierender Frieden setzt primär eine Zusammenarbeit zwischen Israel, Jordanien und einem zukünftigen Staat Palästina voraus. In diesem Kontext ist es unerheblich, ob Palästina in eine Konföderation mit Jordanien eingebunden oder selbständig wird. Trotz der Zurückhaltung Jordaniens hinsichtlich der Option einer palästinensisch-jordanischen Konföderation bildete der Handelsvertrag vom 26. Januar 1995 zwischen Jordanien und der "Palestine National Authority" (PNA) einen wichtigen Bau-

Siehe hierzu: George Hawatmeh, New Government in Place, in: Middle East International vom 20. Januar 1995, S. 10.

Für den Friedensvertrag mit Israel stimmten im Unterhaus 55 Abgeordnete; gegen den Vertrag stimmten 23 Mitglieder. Im 40-köpfigen Senat stimmten 33 Senatoren für den Vertrag, vgl. Jordan Times vom 8. und 10. November 1994.

Siehe hier zu den Bericht der Neuen Zürcher Zeitung "Protest gegen den Friedensprozeß in Amman" vom 27.10.1994.

Vgl. Kronprinz al-Hasan, Search for Security in Mideast will be futile unless socio-economic issues are adressed, in: Jordan Times vom 29.-30. September 1994.

Vgl. hierzu Aziz Alkazaz, Ökonomische Aspekte des Nahost-Friedensprozesses, in: Aus Politik und Zeitgeschichte, B 21-22/ 1994, S. 15-20.

Israel favorisiert zur Realisierung eines Nahostmarktes ein dreistufiges Modell. In der ersten Stufe soll eine wirtschaftliche Kooperation zwischen Israel, Ägypten und Jordanien zustande kommen. In der zweiten Stufe sollen gemeinsame regionale Projekte mit Unterstützung der Geberländer (USA, EU und Japan) entstehen. In der dritten Stufe soll die Zusammenarbeit der nahöstlichen Staaten, nach dem Beispiel der europäischen Zusammenarbeit, institutionalisiert werden, vgl. hierzu Middle Eastern Economic Digest vom 25. November 1994, S. 2. 
stein in einem möglichen israelisch-jordanisch-palästinensischen Markt. ${ }^{37}$ Denn die "Declaration of Principles on Interim Self-Government Arrangements" schreibt gemeinsame israelisch-palästinensische Wirtschafts- und Entwicklungsprogramme vor, die die Bereiche Wasserhaushalt, Verkehr, Kommunikation, Finanzfragen, Industrie, Umweltschutz etc. einschließen. Jordanien, das vor dem Friedensprozeß wirtschaftlich mit der Westbank eng verbunden war, hat realpolitisch keine andere Option, als in diesem Rahmen seine Rolle zu suchen. Politisch und rechtlich bildet der jordanisch-israelische Friedensvertrag die Basis für diese Zusammenarbeit.

\section{Das Vertragswerk}

\section{1. Überblick}

Ähnlich wie der ägyptisch-israelische Friedensvertrag vom 26. März 1979 regelt das vorliegende Vertragswerk ${ }^{38}$ nicht nur die mit der Beendigung des Kriegszustandes unmittelbar zusammenhängenden Punkte wie Grenzfestlegung, Truppenabzug, Bewältigung des Flüchtlingsproblems und die mit der Erhaltung des Friedenszustandes verbundenen Sicherheitsfragen, sondern enthält weitergehende Bestimmungen, etwa über die Aufnahme diplomatischer Beziehungen (Art. 5) oder die Zusammenarbeit auf den Gebieten Wirtschaft (Art. 7) sowie Kultur und Wissenschaft (Art. 10). Darüber hinaus gibt es jedoch diverse Absichtserklärungen, die im Vertrag mit Ägypten nicht enthalten sind, etwa über die Kooperation auf Gebieten wie Tourismus (Art. 17), Verbrechens- und Drogenbekämpfung (Art. 12), Straßenbau (Art. 13), Flugverkehr (Art. 15), Telekommunikation (Art. 16), Umweltschutz (Art. 18), Gesundheit (Art. 21) und landwirtschaftliche Entwicklung (Art. 22). Eine ausführliche Regelung hat schließlich das für den gesamten Nahen Osten ständig brisante Thema der Nutzung der raren Wasservorräte in der Region erfahren (Art. 6). Der inhaltliche Umfang der getroffenen Bestimmungen zeigt, daß beide Seiten nicht nur die militärische Bedrohung beenden, sondern einen stabilen Rahmen für ein dauerhaftes Miteinander in der Region schaffen wollten. Jordanien bekräftigt darüber hinaus mit dem Vertragsschluß seine internationale und regionale Neuorientierung, die in einer Lockerung der Beziehungen zu den arabischen Nachbarn und weiterer Hinwendung zum Westen besteht. Dies kommt deutlich zum Ausdruck in der Rede des zu der Zeit amtierenden jordanischen Ministerpräsidenten Abd al-Salam al-Majali vor dem Parlament in Amman vom 7. Dezember $1994,{ }^{39}$ in der es u.a. heißt: "Peace is not just a treaty with

Der Vertrag beschäftigt sich mit den Zoll- und Transitfragen, siehe zum vollständigen Text des Vertrags Jordan Times vom 29. Januar 1995. Vgl. Fn. 1.

Auszüge der Rede in englischer Übersetzung abgedr. in: BBC Summary of World Broadcasts, Part 4, The Middle East, 13. Dez. 1994. 
Israel. It is a complete change of our regional and international role. (...) We fully realize that our role becomes immune and strong if we and our Arab brothers reach agreement on coordination and cooperation formulas that render us more capable of tackling changes and making them serve our common Arab interests. (...) In view of this state of affairs, we seek to turn Jordan's position and its political and geographic location and its resources and those of its people into an asset that enable us to get the maximum possible for Jordan and the Arabs. (...) Arab aid funded by oil is a thing of the past."

Aus der besonderen Rolle, die Jordanien in der Palästina-Frage innehat, folgt, daß einige Regelungen nur im Zusammenhang mit der noch nicht abschließend geklärten Frage des arabisch-israelischen Konflikts verständlich sind. Schon in der Präambel des Vertrages werden die Resolutionen 242 und 338 des Sicherheitsrates der Vereinten Nationen genannt, die beide bereits in Art. 1 der israelisch-palästinensischen "Declaration of Principles on Interim Self-Government Arrangements" ${ }^{40}$ in Bezug genommen worden waren und die nicht nur das Verhältnis zwischen Israel und Jordanien zum Gegenstand haben, sondern den Nahost-Konflikt insgesamt und vor allem die Besetzung palästinensischer Gebiete durch israelische Streitkräfte und den damit zusammenhängenden Flüchtlingsstrom. Auf die Nahost-Problematik beziehen sich besonders die Bestimmungen der Art. 4 Ziff. 4a (Verhältnis zu anderen Staaten) und 5a-c (Terrorismusbekämpfung) sowie die Art. 8 (Flüchtlingsproblem) und 9 (Status Jerusalems).

\section{Grenzen und Wasser}

Art. 3 i.V.m. Annex Ia bestimmt die Grenzen zwischen Israel und Jordanien. Es sind im wesentlichen die von 1967, d.h. Israel räumt die seinerzeit eroberten Gebiete auf jordanischer Seite. Allerdings handelt es sich nur um einen minimalen Grenzstreifen, so daß die Bedeutung der Regelung weniger in der Rückgabe des Gebietes liegt als in der Tatsache, daß nun zum zweiten Mal ein Teil der Grenzen Israels durch einen internationalen Vertrag festgeschrieben wurde. Zum ersten Mal war dies durch den ägyptisch-israelischen Friedensvertrag geschehen, im übrigen war Israel immer mehr oder weniger bemüht, die Grenzfrage offen zu halten. ${ }^{41}$

In dem betreffenden Gebiet leben inzwischen einige wenige israelische Siedlerfamilien, die dort Landwirtschaft betreiben. Der Frage wird Rechnung getragen durch Art. 3 Buchst. h und i i.V.m. Annex Ib und Ic. Dort wird bestimmt, daß die privaten Eigentumsrechte der

40

Text in: International Legal Materials, Vol. XXXII, No 6, Nov. 1993, S. 1525 ff.

41 Siehe näher Claudia Schmid, Der Israel-Palästina-Konflikt und die Bedeutung des Vorderen Orients als sicherheitspolitische Region nach dem Ost-West-Konflikt, Baden-Baden, 1993, S. $121 \mathrm{ff}$. 
israelischen Siedler unangetastet bleiben und daß die Souveränität Jordaniens über dieses Gebiet, die grundsätzlich anerkannt wird, für zunächst 25 Jahre gewissen Einschränkungen unterliegt. Für die Landeigentümer sowie deren Angestellte gelten nicht die üblichen jordanischen Einreisebestimmungen, sondern sie können formlos zu ihrem Grundbesitz gelangen. Einreiseerleichterungen sollen daneben israelische Polizeibeamte erfahren, die zu Strafverfolgungszwecken Zugang zu diesen Gebieten begehren. Israel verpflichtet sich im Gegenzug, den betreffenden Siedlern das Tragen von Waffen zu verbieten, friedensgefährdende Tätigkeiten zu verhindern sowie Abfallanlieferung von außerhalb zu unterbinden. Ferner ist die Frage des anwendbaren Rechts geregelt: Es gilt grundsätzlich jordanisches Recht, allerdings mit einigen Einschränkungen. Keine Anwendung findet jordanisches Strafrecht, soweit nur israelische Staatsangehörige betroffen sind. Es dürfen keine diskriminierenden Steuern und Abgaben für die Siedler erhoben werden, d.h. Jordanien besitzt zwar die Abgabenhoheit, darf aber für das betreffende Gebiet kein Sonderrecht erlassen, sondern nur Entrichtung nach allgemeinem, also auch für jordanische Staatsbürger geltendem, jordanischem Recht verlangen. Schließlich darf Jordanien bezüglich des Grundbesitzes keine besonderen, d.h. nach allgemeinem jordanischen Recht nicht vorgesehenen Verfügungsbeschränkungen erlassen: Die Siedler können also ihr Land belasten, verkaufen, verschenken und vererben, soweit dies auch für Jordanier möglich ist. Allerdings sind Erwerbungen durch nicht-jordanische Staatsangehörige nur bei vorheriger Genehmigung Jordaniens möglich. Bemerkenswert ist weniger der Umfang der Beschränkungen, denen die Anwendung jordanischen Rechts auf dem betreffenden Gebiet unterliegt, sondern vielmehr der Umstand, daß für die israelischen Siedler doch weitgehend jordanisches Recht gelten soll. Bei näherer Betrachtung zeigt sich jedoch, daß die jordanische Rechtsordnung im Vergleich zur israelischen - jedenfalls soweit das Grundstücksrecht betroffen ist keinen erheblichen Unterschieden unterliegt, da beide Rechtsordnungen in diesem Punkt auf dem britischen Mandatsrecht basieren. Da Erb- und Familienangelegenheiten sich nach dem Recht des Heimatstaates richten, ergeben sich für die Siedler keine einschneidenden Änderungen in ihrem rechtlichen Status. Lediglich die jordanische Steuerhoheit stellt einen Risikofaktor dar. Angesichts des geringen Ausmaßes des betroffenen Personenkreises mag es vielleicht erstaunen, da $\beta$ die Frage eine derart detaillierte Regelung erfahren hat. Dies könnte damit zusammenhängen, daß das Problem in größerem Maße bei den weiteren palästinensisch-israelischen Verhandlungen ansteht und bei dieser Gelegenheit die für Israel innenpolitisch durchsetzbaren Lösungslinien vorgezeichnet wurden. In der "Declaration of Principles" war die Frage der "settlements" ausdrücklich späteren Verhandlungen vorbehalten worden ${ }^{42}$, und selbst im "Israel-PLO Agreement on the Gaza Strip and the Jericho Area" vom 4. Mai $1994^{43}$ wurden die israelischen Siedlungen von palästi- 
nensischer Rechtsprechungshoheit weitestgehend ausgenommen ${ }^{44}$, so daß die Frage einer endgültigen Klärung noch harrt. ${ }^{45}$ Angesichts des zunehmenden Widerstandes der israelischen Siedler in den besetzten Gebieten gegen eine palästinensische Autonomie sind alle Fragen, die mit dem zukünftigen Schicksal der Siedlungen außerhalb des israelischen Kernlandes zusammenhängen, von besonderer Brisanz, so daß eine laxe Behandlung auch weniger Fälle zu heftigen Reaktionen führen kann.

Art. 6 des Vertrages i.V.m. der Anlage II regelt die Aufteilung der raren Wasserressourcen zwischen Israel und Jordanien, eine Frage, die für die Beteiligten von existenzieller Bedeutung ist. Der Streit um das Wasser des Jordan sowie seiner Quellflüsse Yarmuk, Banias und Hasbani zwischen Syrien, Jordanien und dem Libanon sowie Israel begann schon in den fünfziger Jahren. 1955 legte ein Berater des amerikanischen Präsidenten Eisenhower den nach ihm benannten Johnston-Plan vor, der eine Quotenregelung bezüglich der Wasserentnahme enthielt. ${ }^{46}$ Dieser wurde jedoch von allen Beteiligten abgelehnt. In den Jahren 1953-1965 realisierte Israel sein Projekt "National Water Carrier", durch das es mindestens 50 Prozent des Jordanwassers umleitete. Daraufhin beschloß die Arabische Liga auf der Gipfelkonferenz im Januar 1964 in Kairo - auf der auch die PLO ins Leben gerufen wurde -, daß militärische Aktionen gegen israelische Pumpstationen legitim seien, und gründete die "Jordan Water Organization", die die Realisierung eines eigenen Wasserableitungsprojektes zum Ziel hatte. ${ }^{47}$ Die diesbezüglichen Bauarbeiten wurden von den israelischen Streitkräften attackiert, und die nunmehr gewaltsamen Auseinandersetzungen um das Wasser mündeten letztlich im Sechs-Tage-Krieg. ${ }^{48}$ In diesem Vertrag geht es um die Flüsse Jordan und Yarmuk sowie die Grundwasservorräte im Wadi Araba. Die Bestimmung enthält eine detaillierte Regelung, die die Position Jordaniens vor allem bezüglich des Yarmuk stärkt ${ }^{49}$, aber darüber hinaus eine ausgedehnte Zusammenarbeit zwischen den Parteien bezüglich ehrgeiziger Wasserprojekte vorsieht, die mit internationaler Unterstützung verwirklicht werden sollen. ${ }^{50}$ Die Vertragsparteien sind jedoch nicht die einzigen, die an den Wasservorkommen Anteil beanspruchen und nach allgemeinem Völkerrecht auch

Art. V Nr. 1a, Annex III Art. I und III.

Zu Entwicklung und Stand der komplizierten Rechtslage in den besetzten Gebieten s. Ludwig Watzal, Frieden ohne Gerechtigkeit? Israel und die Menschenrechte der Palästinenser, Köln 1994, S. $58 \mathrm{ff}$.

Manuel Schiffler, Nachhaltige Wassernutzung in Jordanien. Determinanten, Handlungsfelder und Beiträge der Entwicklungszusammenarbeit, Berlin: Deutsches Institut für Entwicklungspolitik 1993, S. $61 \mathrm{f}$.

Claudia Schmid, a.a.O., S. 106 f.; Mark W. Zacher, International Conflicts and Collective Security, 1946-77, New York usw., 1979, S. 166.

Claudia Schmid, a.a.O., S. 107. 
diesbezügliche Rechte haben ${ }^{51}$. Zu beteiligen sind auch Syrien und die Palästinenser ${ }^{52}$, so da $\beta$ auf lange Sicht nur eine multilaterale Regelung ein funktionierendes Wasserregime installieren kann.

\section{Das Verhältnis zu anderen arabischen Staaten}

In diesem Zusammenhang ist Art. 4 Ziff. 4a von besonderem Interesse, der beiden Parteien jegliche Zusammenarbeit mit Koalitionen oder Organisationen militärischen oder sicherheitspolitischen Charakters verbietet, dessen Ziele Feindseligkeiten gegen die andere Partei einschließen. Diese Bestimmung könnte vor allem Jordanien wegen seiner Mitgliedschaft in der Arabischen Liga in völkerrechtliche Konflikte bringen, da dieser Zusammenschluß arabischer Staaten, der 1945 von Ägypten, Irak, Jordanien, Libanon, Saudi-Arabien, Syrien und dem Jemen gegründet wurde, in Annex 1 seiner Satzung ${ }^{53}$ die Unabhängigkeit Palästinas als arabische Nation bekräftigt und damit die Existenzberechtigung Israels bestreitet Ägypten wurde 1979 wegen seines Friedensschlusses mit Israel ausgeschlossen. Ferner hat die Liga eine nicht unbedeutende sicherheitspolitische Komponente. Art. 6 der Satzung bestimmt: "In case of aggression or threat of aggression by one state against a member state, the state which has been attacked or threatened with aggression may demand the immediate convocation of the Council. The Council shall by unanimous decision determine the measures necessary to repulse the aggression. (...)" Eine Definition, wann eine Aggression vorliegt, enthält die Satzung nicht, so daß der Council im Einzelfall auch hierüber befinden muß. Theoretisch könnte also die Liga ihre Mitglieder verpflichten, gegen Israel militärisch vorzugehen. Da jedoch der Council in diesem Falle nur einstimmig beschließen kann, ist eine entsprechende Entscheidung gegen die Stimme Jordaniens nicht möglich. 1950 beschloß der Council der Arabischen Liga jedoch den "Treaty of Joint Defence and Economic Co-operation Among the States of the Arab League" ${ }^{54}$, der im gleichen Jahr von

So schon die Helsinki-Regeln der International Law Association von 1966; s. ferner Johan G. Lammers, Pollution of International Watercourses, Boston usw., 1984, S. $556 \mathrm{ff}$;; Christian Gloria, in: Knut Ipsen, Völkerrecht, 3. Aufl., München, 1990, § 23 Rn. 83 f.; auch der Entwurf der International Law Commission der VN von 1991 (abgedr. in: Yearbook of the International Law Commission 1991 Vol. II (Part Two), S. 66 ff.) sieht in Art. 5 vor, daß grenzüberschreitende Wasserquellen angemessen auf zuteilen sind ("equitable and reasonable utilization and participation"). Zum ganzen ausführlich Patricia W. Birnie / Alan E. Boyle, International Law and the Environment, Oxford, 1992, S. 215 ff. m.w.N.

52

$\mathrm{Zu}$ deren Ansprüchen s. etwa die Jordan Times vom 3.12.1994. In der Declaration of Principles (Annex III Nr. la) bzw. dem Gaza-Jericho-Agreement (Annex II Art. II Nr. 31 und 35) ist die Wasserfrage nur ansatzweise geregelt.

UN Treaty Series Vol. 70, p. 237. Die englische Fassung ist ferner abgedr. in: Ahmed M. Gomaa, The Foundation of The League of Arab States, Bungay (Suffolk), 1977, S. 295 ff.

54 Abgedr. in: Muhammad Khalil (Hrsg.), The Arab States and The Arab League, Vol. II (International Affairs), Beirut 1962, Doc. 43, S. 101 ff. Einen Überblick über Funktion und Organe gibt 
den Mitgliedstaaten angenommen wurde. Dieser Beistandspakt wurde gegründet, um Angriffe nicht-arabischer Staaten, sprich von Seiten Israels, gemeinsam abzuwehren. ${ }^{55}$ Art. 6 konstituiert den "Joint Defence Council", der lediglich einer Zweidrittelmehrheit bedarf, um Maßnahmen zu beschließen, die für die Mitglieder bindend sind. Die Ermächtigung bezieht sich insbesondere auf Maßnahmen der individuellen und kollektiven Selbstverteidigung (Art. 2). Allerdings ist für die Kontrolle des Joint Defence Council der Council der Liga zuständig, der nur einstimmig beschließen kann. Aber hier wirkt sich gerade das Einstimmigkeitsprinzip als Behinderung des Council der Liga in seiner Kontrollfunktion gegenüber dem Joint Defence Council aus. Jordanien kann also theoretisch gegen seine Stimme zu militärischen Maßnahmen verpflichtet werden. ${ }^{56}$ Dies dürfte jedoch nach der derzeitigen politischen Lage nur von geringer praktischer Bedeutung sein. Art. 25 Ziff. 2 des vorliegenden Vertrages besagt immerhin, daß beide Parteien der Auffassung sind, da $\beta$ die sich hieraus ergebenden Pflichten nicht unvereinbar mit bereits bestehenden Verpflichtungen beider sind. Im Zweifel sollen die Pflichten aus diesem Vertrag Vorrang haben. Diese Bestimmung hat allerdings keinen Einfluß auf Jordaniens Verpflichtungen gegenüber anderen arabischen Staaten und kann den rechtlichen Konflikt daher nicht lösen.

\section{Das Terrorismusproblem}

Art. 4 Ziff. 5 bestimmt, daß beide Parteien effektive Maßnahmen zu treffen haben, um den Terrorismus zu bekämpfen, insbesondere zu verhindern, daß von ihrem Staatsgebiet aus terroristische Aktionen durch Duldung der direkten Vorbereitung oder der Bildung entsprechender Organisationen ihren Ausgangspunkt nehmen; ferner wird Zusammenarbeit gegen grenzüberschreitende "Infiltration" vereinbart. Diese Regelung dürfte auf radikale und islamistische Gruppierungen, wie das General Command der PFLP (Popular Front for the Liberation of Palestine) unter Ahmed Jibril sowie die Hamas (Harakat al-muqawama alislamiya = Bewegung des islamischen Widerstandes) und die "Islamischer-Gihad"-Gruppe, zielen, die den Verhandlungskurs der PLO zu unterminieren versuchen. Allerdings kann weder Jordanien Interesse daran haben, seine nunmehr offen vertretene Kooperationslinie mit den westlich orientierten Staaten durch die mehr oder weniger offene Unterstützung von Terroristen mit radikal antiwestlichen Zielen selbst zu untergraben, noch dürfte Israel weiterhin versucht sein, etwa die Hamas geheimdienstlich zu unterstützen, um die PLO zu schwächen. Der Regelung kommt somit weniger praktische Bedeutung als vielmehr Signal-

auch Mufeed Shihab, in: Rudolf Bernhardt (Hrsg.), Encyclopedia of Public International Law (im folgenden: EPIL) Bd. 6, Amsterdam ,1983, "Arab States, League of", S. 20 (23 f.); s. ferner Robert W. MacDonald, The League of Arab States. A Study in the Dynamics of Regional Organization, Princeton, 1965, S. 226 ff.

56

Mark W. Zacher, a.a.O., S. 166.

Anderer Ansicht Mark W. Zacher, ebd. 
funktion zu, so daß es müßig wäre, die besondere Problematik von Durchsetzung und Kontrolle solcher Bestimmungen zu erörtern. Jedenfalls erscheint es ausgeschlossen, daß die angestrebte Zusammenarbeit irgendeine Einschränkung von Souveränitätsrechten beinhalten soll, also etwa Interventionen bzw. gewaltsame Aktionen auf dem Nachbargebiet vergleichbar mit den israelischen Operationen im Libanon - zu rechtfertigen vermag. Es sei daher lediglich auf Art. 3 Ziff. 6 hingewiesen, der die Einrichtung eines Überwachungsund Konsultationsmechanismus für alle Sicherheitsfragen vorsieht, ohne jedoch die Besetzung entsprechender Gremien oder auch nur die Beteiligung neutraler Beobachter im einzelnen anzusprechen.

\section{Die Flüchtlingsfrage}

Art. 8 des Vertrages behandelt die Frage der "Refugees and Displaced Persons", gemeint sind die Palästinenser. Zunächst wird festgestellt, daß die menschlichen Probleme, die aus dem Nahostkonflikt resultieren auf bilateraler Ebene nicht völlig gelöst werden können. Dies mag dem Grunde nach richtig sein, da auch Ägypten, Syrien und der Libanon von der Frage betroffen sind und Regelungen zu Lasten dritter Staaten rechtlich nicht möglich sind. ${ }^{57}$ Dennoch hätte man vielleicht erwarten können, daß die Problematik, die ausschließlich die vertragschließenden Staaten betrifft, d.h. die Frage der Rückkehr von Palästinensern aus Jordanien, im Vertrag eine konkrete Regelung erfahren. Aber auch eine darauf beschränkte Regelung wäre im Hinblick auf den Verhandlungsprozeß zwischen Israel und der PLO problematisch gewesen und hätte den dortigen Ergebnissen vorgegriffen. Die viel erörterte Frage, ob und welche Palästinenser das Recht haben, in ihre angestammte Heimat zurückzukehren $^{58}$, wurde daher völlig ausgespart. Man hat sich vielmehr mit einer Bestimmung begnügt, die keinerlei sachliche Regelung enthält, sondern lediglich die Möglichkeit, wie die Frage weiter zu behandeln ist, grob vorzeichnet, nämlich durch die Bildung multilateraler Gremien, und zwar vor allem unter Beteiligung Ägyptens und der Palästinenser. Auch hier gilt allerdings, daß die Verwirklichung dieser Bestimmung von der Mitarbeit der Genannten und eventuell anderer, etwa der USA, der EU oder der UNO, abhängig bleibt. Die insgesamt mehr technische als inhaltliche Regelung entspricht auch

Vgl. etwa Art. 34 und 35 der Wiener Vertragsrechtskonvention vom 23.05.1969 (BGBl. 1985 II, 926); ferner Alfred Verdroß / Bruno Simma, Universelles Völkerrecht, 3. Aufl., Berlin, 1984, § 764; Wolff Heintschel von Heinegg, in: Knut Ipsen, Völkerrecht, 3. Aufl., München, 1990, § 12 Rn. 29, 32; Ian Brownlie, Principles of Public International Law, 4. Aufl., Oxford, 1990, S. 622 f.

S. hierzu etwa Kurt R. Radley, The Palestinian Refugees: The Right to Return in International Law, American Journal of International Law Vol. 72 (1978), S. 586 ff.; Alfred-Maurice de Zayas, in: Rudolf Bernhardt (Hrsg.), EPIL Bd. 8, Amsterdam, 1985, "Population, Expulsion, Transfer", S. 438 (442), jeweils m. Nachw. zu entsprechenden Resolutionen der Generalversammlung der VN. 
der Arbeit der "Refugee Working Group", die auf der Madrider Konferenz 1991 gegründet wurde und sich bisher ebenfalls nicht mit der Frage des Rückkehrrechts befaßt hat.

Die Bestimmung trifft eine Unterscheidung zwischen "Refugees" und "Displaced Persons". Während für die Gruppe der "Displaced Persons" ein vierköpfiges Komitee gebildet werden soll, an dem Israel, Jordanien, Ägypten und ein Vertreter der Palästinenser beteiligt sein sollen, wird für die "Flüchtlinge" eine wesentlich vagere Behandlung vorgesehen im Rahmen der Arbeit der "Multilateral Group on Refugees" und in Gesprächen im Zusammenhang mit den Verhandlungen über die besetzten Gebiete. Eine ähnliche Behandlung der Frage wurde bereits in der israelisch-palästinensischen "Declaration of Principles" in Aussicht genommen, in der in Art. XII die Bildung eines israelisch-palästinensischen Komitees unter Beteiligung Ägyptens und Jordaniens vorgesehen wurde, um die Problematik der 1967 vertriebenen ("displaced") Palästinenser zu behandeln, während die Frage der Flüchtlinge ("Refugees"), die bereits in der Zeit bis 1948 ihre Heimat verlassen haben, zunächst ungeklärt bleibt ${ }^{60}$ und im Zusammenhang mit den endgültigen Statusfragen geklärt werden soll. ${ }^{61}$ Gerade hier zeigt sich besonders deutlich der enge Zusammenhang des israelischjordanischen Friedensvertrages mit den Friedensverhandlungen zwischen Israel und den Palästinensern sowie das Bemühen aller Beteiligten, beide Prozesse auf einander abzustimmen.

Die strikte Trennung im vorliegenden Vertragstext des Art. 8 zwischen "Refugees" und "Displaced Persons" entspricht allerdings nicht dem inzwischen üblichen Sprachgebrauch. Der Begriff Flüchtling ("Refugee") im strengen Sinne des Wortes wurde in Art. 1 A Nr. 2 der Flüchtlingskonvention vom 28.07.1951 ${ }^{62}$ definiert als eine Person, "die aus der begründeten Furcht vor Verfolgung wegen ihrer Rasse, Religion, Nationalität, Zugehörigkeit zu einer bestimmten sozialen Gruppe oder wegen ihrer politischen Überzeugung sich außerhalb des Landes befindet, dessen Staatsangehörigkeit sie besitzt, und den Schutz dieses Landes nicht in Anspruch nehmen kann oder wegen dieser Befürchtungen nicht in Anspruch nehmen will; oder die sich als staatenlos infolge solcher Ereignisse außerhalb des Landes befindet, in welchem sie ihren gewöhnlichen Aufenthalt hatte, und nicht dorthin zurückkehren kann oder wegen der erwähnten Befürchtungen nicht dorthin zurückkehren will." Für die humanitäre Zielsetzung der Konvention erwies sich diese Definition alsbald als zu eng, so daß der Begriff "Displaced Persons" sich für all die jenigen etablierte, die sich

59

60

61

62

Hierzu Elia Zureik, Journal of Palestine Studies XXIV, No 1 (1994), S. 5 (7).

Vgl. Art. V Ziff. 3 der Deklaration.

Elia Zureik, a.a.O.; Art. XVI des Gaza-Jericho-Agreements hat diese Bestimmung unverändert übernommen.

BGBl. 1953 II, 559 (geändert 31.01.1967, BGBl. 1969 II, 1293): Die Konvention hat über hundert Mitglieder, so daß die dortige Definition als allgemein anerkannt gelten kann, s. Eberhard Jahn, in: Rudolf Bernhardt (Hrsg.), EPIL Bd. 8, Amsterdam, 1985, "Refugees", S. 452 (453). 
tatsächlich in einer den Flüchtlingen vergleichbaren Lage befinden, obwohl sie die Staatsangehörigkeit eines Staates besitzen, von dem sie nicht verfolgt werden, der ihnen jedoch aufgrund der tatsächlichen Umstände keinen Schutz und keine Hilfestellung bei der Rückkehr in ihre Heimat bieten kann. ${ }^{63}$ In einer großen Anzahl von Resolutionen der Generalversammlung der UNO sowie des UN-Hochkommissars für Flüchtlinge werden daher beide Begriffe unterschiedslos verwendet. ${ }^{64}$ Auch statistische Angaben, die die Situation der Palästinenser betreffen, unterscheiden nicht zwischen Refugees und Displaced Persons. ${ }^{65}$

Es bleibt somit die Frage, welche Bedeutung die Unterscheidung im vorliegenden Vertragstext hat. Flüchtlinge im vorgenannten engen Sinne sind nur diejenigen, die entweder keine Staatsangehörigkeit besitzen oder die aus Furcht vor Verfolgung die Hilfe ihres Staates bei der Rückkehr nicht in Anspruch nehmen. Ersteres trifft lediglich auf diejenigen Palästinenser zu, die vor 1950 Palästina verlassen haben und die nicht nach Jordanien gezogen sind; denn von 1950 bis 1988 beanspruchte Jordanien die Westbank als eigenes Staatsgebiet und verlieh allen Palästinensern, die sich im Lande selbst oder in der Westbank aufhielten, die jordanische Staatsbürgerschaft. Diese haben sie auch nach 1988 bis auf weiteres inne. Lediglich diejenigen, die damals in andere arabische Staaten wie Ägypten, Libanon, Syrien und Libyen geflohen sind, sind bis heute staatenlos. Dasselbe gilt für die Palästinenser im Gazastreifen, den zunächst Ägypten verwaltete, ohne jedoch den Einwohnern die ägyptische Staatsbürgerschaft zu verleihen. Letztere haben jedoch ganz überwiegend ihre Heimat bis heute nicht verlassen, so daß sie von der Frage des Rückkehrrechts nicht betroffen sind. Flüchtlinge im engen Sinne des Wortes sind somit lediglich die Vertriebenen von 1948/49, die nicht Jordanier geworden sind. Alle in Jordanien lebenden Palästinenser sowie alle diejenigen, die erst 1967 die Westbank - ganz gleich wohin verlassen haben, sind jordanische Staatsangehörige und fallen daher unter den Begriff Displaced Persons. Dementsprechend werden die bereits bis 1948 vertriebenen Palästinenser in der Resolution der Generalversammlung der VN 194 vom 11. Dezember 1948 als "Refugees" bezeichnet, während die Resolution 3236 aus dem Jahre 1974 von Palästinensern spricht, die "displaced and uprooted" sind.

Es erscheint jedoch fraglich, ob die Regelung eine solch gezielte Unterscheidung beabsichtigt oder nicht vielmehr in Anlehnung an die oben genannte israelisch-palästinensische Declaration of Principles mit Refugees die Flüchtlinge von 1948/49 meint und mit Displaced Persons die Vertriebenen von 1967, zumal auch viele Palästinenser ihre jordani-

Eberhard Jahn, a.a.O., S. 453; s. Aga Khan, Legal Problems Relating to Refugees and Displaced Persons, Recueil des Cours 149 (1976 I), S. 287 (295 f.).

64

Eberhard Jahn, a.a.O., S. 453 m.Nachw.

65

Vgl. etwa die Map of UNRWA's Areas of Operations vom Juni 1991, abgedr. bei Elia Zureik, a.a.O., S. 6; s. ferner die undifferenzierte Verwendung des Begriffs "Refugee" in demselben Beitrag, passim. 
sche Staatsangehörigkeit aus Furcht vor Verfolgung in Jordanien aufgrund der Unruhen 1970/71 nicht in Anspruch genommen haben und im Einzelfall die Rechtslage nur schwer feststellbar sein wird.

\section{Der Status Jerusalems}

Art. 9 befaßt sich mit den Stätten von historischer und religiöser Bedeutung, womit die islamischen Heiligtümer in Jerusalem gemeint sind, d.s. die al-Aqsa-Moschee und der Felsendom sowie die religiösen Stiftungen (awqaf) mit ihren Besitztümern. Diese befinden sich ausschließlich auf dem Gebiet Ost-Jerusalems, das von Israel 1967 besetzt wurde, während der westliche Teil der Stadt bereits seit 1948 unter israelischer Herrschaft steht und von der Regelung nicht berührt wird. Art. 9 Ziff. 2 bestätigt die "gegenwärtige spezielle Rolle" Jordaniens bezüglich dieser Heiligtümer und verpflichtet Israel auf eine auch zukünftige Berücksichtigung dieser Rolle bei etwaigen Verhandlungen über den Status Jerusalems, Verhandlungen also mit der PLO, die neben den Gebietsansprüchen auf OstJerusalem ihrerseits die Aufsicht über die Heiligtümer anstrebt. ${ }^{66}$

Die Regelung hat denn auch zu negativen Reaktionen auf palästinensischer Seite und einem Disput zwischen König Hussein und Arafat geführt. ${ }^{67}$ Jordanien hat sich mit dem Argument verteidigt, die Bestimmung in dieser Art und Weise sei juristisch notwendig gewesen, um eine Festigung der Position Israels zu verhindern, solange es noch keinen palästinensischen Souverän gebe. Die PLO hingegen ist der Auffassung, daß die mit Israel getroffene Vereinbarung mangels palästinensischer Präsenz einer Hebräisierung des arabischen Teils Jerusalems Vorschub leiste, und vermutet die versteckte Absicht Jordaniens, sich die Hoheit über die Heiligtümer auf Dauer zu sichern. ${ }^{68}$ Schon Mitte Dezember 1994 war König Hussein auf dem Gipfeltreffen der Islamic Conference Organization in Casablanca mit seinem Anliegen, die Rolle Jordaniens in bezug auf Jerusalem festzuschreiben, nicht durchgedrungen ${ }^{69}$ und daraufhin abrupt abgereist. ${ }^{70}$

Der völkerrechtliche Status jedenfalls Ost-Jerusalems zeigt, daß die Position Jordaniens zumindest zweifelhaft ist. Sie basiert auf der Annahme, daß Israel, das Ost-Jerusalem während des Sechs-Tage-Krieges 1967 besetzt hat, ein Annexionsrecht unter Ausschluß

Die Frage Jerusalems wurde in Art. V Nr. 3 der Declaration of Principles ausdrücklich weiteren Verhandlungen vorbehalten.

67 Jordan Times vom 26.01.1995.

68 Ebd.

69 70 BBC Summary of World Broadcasts, Part 4, The Middle East, 19. Dez. 1994, S. 4. Jerusalem Post vom 15.12.1994. 
aller arabischen Rechte erwächst, sobald Jordanien seinen Anspruch auf die Heiligtümer Jerusalems aufgibt. Es ist jedoch allgemein anerkannt, daß militärische Eroberungen zumindest in der Zeit nach dem Zweiten Weltkrieg in keinem Fall zu einer Annexion des eroberten Gebietes berechtigen. ${ }^{71}$ Für Ost-Jerusalem gilt daher dasselbe wie für die besetzten Gebiete ${ }^{72}$, und dasselbe muß auch gelten für die Hoheit über Heilige Stätten. Wegen seiner großen Bedeutung für drei Weltreligionen ist Jerusalem seit Jahrhunderten der Zankapfel nicht nur der nahöstlichen Staaten. So beansprucht etwa der Vatikan bis heute gewisse Rechte bezüglich der heiligen Stätten Jerusalems, soweit sie für die Christen Bedeutung haben, und verweigerte aus diesem Grunde dem Staate Israel zunächst die Aufnahme diplomatischer Beziehungen. ${ }^{73}$ Mehrfach wurde ohne Erfolg vorgeschlagen, ganz Jerusalem einem internationalen Regime zu unterstellen. ${ }^{74}$ Die Brisanz des Themas führte dazu, daß die Frage nach dem Status Jerusalems bei Friedensverhandlungen bisher außen vor gelassen wurde, denn einerseits steht eine Auf gabe West-Jerusalems durch Israel nicht ernsthaft zur Debatte, andererseits würde eine Beschränkung der Verhandlungen auf den östlichen Teil der Stadt die Anerkennung der Teilung Jerusalems implizieren, zu der sich weder die Konfliktparteien noch andere Staaten bisher haben durchringen können. Auch die Resolutionen des Sicherheitsrates der $\mathrm{VN}^{75}$ beschränken sich auf die Feststellung, daß Maßnahmen Israels den Status Jerusalems nicht zu ändern vermögen, nehmen aber zur Zukunft der Stadt nicht Stellung. Die Lösung der Frage bleibt daher gekoppelt an den Status von Westbank und Gaza und einer entsprechenden Vereinbarung zwischen Israel und den Palästinensern vorbehalten. Auch wenn Israel sich verpflichtet hat, bei den weiteren Verhandlungen die Rolle Jordaniens zu berücksichtigen, ist wohl kaum davon auszugehen, daß Israel eine Einigung mit den Palästinensern an dieser Frage scheitern lassen würde.

Art. 9 des vorliegenden Vertrages ist deswegen mehr von politischer denn rechtlicher Bedeutung in bezug auf den Status Jerusalems, über den Jordanien nicht allein verfügen kann. Die getroffene Regelung deutet darauf hin, daß König Hussein und der israelische Ministerpräsident Rabin sich wohl darüber einig waren, daß die israelische Herrschaft über

S. etwa Alfred Verdroß / Bruno Simma, a.a.O., §§ 971, 1163; Christian Gloria, a.a.O., § 23 Rn. 43, auch speziell zu Jerusalem.

Zu den unterschiedlichen Rechtspositionen der Konfliktparteien sowie anderer Staaten und den VN s. Peter Malanczuk, in: Rudolf Bernhardt (Hrsg.), EPIL Bd. 12, Amsterdam, 1990, "Jerusalem", S. 184 (190 ff.); Jochen Abr. Frowein / Stefan Oeter, Ost-Jerusalem und das deutschisraelische Sozialversicherungsabkommen, Zeitschrift für ausländisches öffentliches Recht und Völkerrecht 1988, S. 18 (22 ff.).

S. hierzu etwa Peter Malanczuk, a.a.O. , S. 191

Ebd., S. $186 \mathrm{ff}$.

S. etwa die Resolutionen des Sicherheitsrates 252 vom 21.05.1968; 267 vom 03.07.1969; 271 vom 15.09. 1969; 298 vom 25.09.1971; 338 vom 22.10 .1973 ; 381 vom 30.11.1975. 
Ost-Jerusalem für Israel nicht zur Disposition steht und eine Übergabe an die Palästinenser damit ausgeschlossen ist. Die Frage nach der Hoheit über die Heiligtümer ist damit allerdings nicht entschieden. Jordanien will seinerseits aus dieser Situation Nutzen ziehen und seine Bedeutung in der Region ausbauen; speziell König Hussein könnte dadurch die innenpolitische Legitimationsbasis seines Königshauses festigen, nachdem die Haschemiten 1924 mit dem Hidschas die Hoheit über die höchsten islamischen Heiligtümer in Mekka und Medina an die saudische Dynastie verloren haben.

\section{Fazit und Ausblick}

Es steht außer Zweifel, daß der jordanisch-israelische Friedensvertrag ein Meilenstein auf dem Weg zu einem umfassenden Frieden im Nahen Osten ist. Jordanien hatte zwar seit der Abkoppelung der Westbank 1988 keine unlösbaren bilateralen Streitigkeiten mit Israel. Es verblieben jedoch zwei Hauptprobleme im Vorfeld der Verhandlungen, die die jordanische Führung durch ihr pragmatisches Vorgehen lösen konnte. Zum einen gelang es König Hussein, sich durch den von ihm eingeleiteten Demokratisierungsprozeß eine notwendige innen- und außenpolitische Legitimation zu verschaffen; zum anderen befreite sich Jordanien Schritt für Schritt von der gesamtarabischen Position, insbesondere von der Verquickung der eigenen Interessen mit denen der Palästinenser. Letztes entsprang der Erkenntnis, daß eine gesamtarabische Strategie zur Beilegung des Nahost-Konflikts nur schwer durchsetzbar war. Syrien allerdings stand infolge seines Anspruchs auf regionale Hegemonie Teillösungen des Nahost-Konflikts, insbesondere bilateralen Abkommen ablehnend gegenüber. Es attackierte daher sowohl das Abkommen zwischen der PLO und Israel als auch den jordanisch-israelischen Friedensvertrag. Erleichtert wurde dagegen der israelisch-jordanische Frieden durch die Prinzipienerklärung zwischen der PLO und Israel. Die Unterzeichnung dieses Abkommens war für Jordanien mit seiner mehrheitlich palästinensischen Bevölkerung von enormer Relevanz. Der Frieden mit Israel konnte nun, nachdem die PLO Frieden mit Israel geschlossen hatte, nicht als Alleingang angesehen werden.

Die weiteren Perspektiven des jordanisch-israelischen Friedens sind von der Entwicklung der regionalen Struktur abhängig. Vor allem in drei Problemfeldern scheint keine Lösung möglich zu sein ohne regionale Zusammenarbeit.

Dies ist zunächst die endgültige Lösung der Flüchtlingsfrage, die sowohl die Vereinbarung zwischen Israel und der PLO als auch der jordanisch-israelische Friedensvertrag nur in vagen Umrissen behandeln. Auch wenn die Frage der Repatriierung der palästinensischen Flüchtlinge aus Jordanien eher eine theoretische Frage ist, weil diese sich nach mehreren Jahrzehnten dort verwurzelt haben, steht eine rechtliche Klärung des Rückkehrrechts nach wie vor an, die auch durch eine Entschädigungsregelung erreicht werden könnte. 
Zum anderen ist die Form der zukünftigen jordanisch-palästinensischen Beziehungen noch offen. Wahrscheinlich wird König Hussein seinen Plan von 1972 zur Bildung eines "Vereinigten Arabischen Königreiches", das Jordanien und die von Israel besetzten Gebiete umfassen sollte, nicht mehr aus der Schublade ziehen. Weder die Palästinenser noch die Jordanier scheinen diese Lösung zu favorisieren. Die wirtschaftlichen und politischen Strukturen des West jordanlands könnten Jordanien destabilisieren.

Das dritte Problemfeld, nämlich die wirtschaftliche Entwicklung und die regionale wirtschaftliche Zusammenarbeit, ist ohne jeden Zweifel grundlegend für die Stabilität der jordanisch-israelischen Beziehungen und einer erfolgreichen Fortsetzung des Friedensprozesses. Trotz der - zögernden - Aufhebung der Boykottierung Israels durch einige arabische Staaten erscheint der Weg bis zur Entstehung eines Nahost-Marktes noch lang und steinig. Aus diesem Grunde ist es offensichtlich, daß keine Alternative zu einer israelisch-palästinensisch-jordanischen Wirtschaftskooperation existiert. Da aber die Jordanier, wie die anderen arabischen Staaten nicht unbegründet Ängste vor der Dominanz der leistungsstarken israelischen Wirtschaft haben, könnte die Internationalisierung der Wirtschaftsprojekte Abhilfe schaffen, zumal Israel, Jordanien und die besetzten Gebiete ohnehin, auch wenn in unterschiedlichem Grad, auf externe Wirtschaftshilfe angewiesen sind. Eine vitale Frage bleibt darüber hinaus die Verteilung der Wasserressourcen, deren Lösung entsprechend den völkerrechtlichen Leitlinien nur durch ein multilaterales Wasserregime zu erreichen ist, durch das nicht nur die Anteile der berechtigten Staaten festgelegt werden sollten, sondern auch gemeinsame Entwicklungsprojekte vereinbart werden können. Insbesondere für eine finanzielle Hilfe von Seiten westlicher Staaten ist eine verbindliche Einigung unter Berücksichtigung aller berechtigten Interessen grundlegende Voraussetzung.

Solange diese Problemfelder nicht befriedigend gelöst worden sind, sind Rückschläge zu erwarten. Dennoch gibt es, und dies ist den Führern der Staaten der Region nach den Umbrüchen in den Strukturen der internationalen Politik und aus wirtschaftlichen Gründen sehr bewußt, keine Alternative zum Frieden. 


\section{The Peace Treaty between Jordan and Israel}

\section{By Irene Schlünder and Ferhad Ibrahim}

Although Jordan intended to solve the conflict with Israel soon after the conference of Madrid, due to internal political and economic necessities, three years had to pass before the Israeli-Jordan peace settlement could be signed in October 1994. Prior to that the PLO had paved the way to peace between Israel and Jordan by signing the Declaration of Principles on Oslo.

On a bilateral basis Israel and Jordan had hardly any disagreements. Their contract solved territorial problems to the satisfaction of both countries. Nevertheless, one important complex of problems remained untouched: the question of palestinian refugees of 1948 and 1967 who settled down in Jordan. The solution of this problem will determine the future development of the Israel-Jordan relationship. 